\title{
Article \\ Aflibercept Plus FOLFIRI as Second-Line Treatment for Metastatic Colorectal Cancer: A Single-Institution Real-Life Experience
}

\author{
Daniele Lavacchi ${ }^{1}$, Giandomenico Roviello ${ }^{2}$, Elisa Giommoni ${ }^{1}$, Lorenzo Dreoni ${ }^{1} @$, Silvia Derio ${ }^{1}$, \\ Marco Brugia ${ }^{1}$, Amedeo Amedei ${ }^{3}{ }^{\circledR}$, Serena Pillozzi ${ }^{1}\left(\mathbb{D}\right.$ and Lorenzo Antonuzzo ${ }^{1,3, *}$ \\ 1 Clinical Oncology Unit, Careggi University Hospital, 50134 Florence, Italy; \\ lavacchid@aou-careggi.toscana.it (D.L.); giommonie@aou-careggi.toscana.it (E.G.); \\ lorenzodreoni@gmail.com (L.D.); silviaderio@yahoo.com (S.D.); brugiamarco@gmail.com (M.B.); \\ serena.pillozzi@unifi.it (S.P.) \\ 2 Department of Health Science, University of Florence, 50134 Florence, Italy; giandomenico.roviello@unifi.it \\ 3 Department of Experimental and Clinical Medicine, University of Florence, 50134 Florence, Italy; \\ amedeo.amedei@unifi.it \\ * Correspondence: lorenzo.antonuzzo@unifi.it
}

Citation: Lavacchi, D.; Roviello, G.; Giommoni, E.; Dreoni, L.; Derio, S.; Brugia, M.; Amedei, A.; Pillozzi, S.; Antonuzzo, L. Aflibercept Plus FOLFIRI as Second-Line Treatment for Metastatic Colorectal Cancer: A Single-Institution Real-Life Experience. Cancers 2021, 13, 3863. https://doi.org/10.3390/ cancers13153863

Academic Editor: Abdolrahman Shams Nateri

Received: 6 July 2021

Accepted: 27 July 2021

Published: 31 July 2021

Publisher's Note: MDPI stays neutral with regard to jurisdictional claims in published maps and institutional affiliations.

Copyright: (c) 2021 by the authors. Licensee MDPI, Basel, Switzerland. This article is an open access article distributed under the terms and conditions of the Creative Commons Attribution (CC BY) license (https:/ / creativecommons.org/licenses/by/ $4.0 /)$.
Simple Summary: The continuum of care for mCRC might include anti-angiogenic drug as antiVEGF/VEGFR moAb and recombinant proteins in combination with fluoropyrimidine-based regimens in first- and second-line treatment, and multikinase inhibitors in refractory patients. The addition of aflibercept to FOLFIRI has been demonstrated to improve survival in patients with metastatic colorectal cancer ( $\mathrm{mCRC}$ ) who progressed after receiving a standard oxaliplatin-based regimen. In this retrospective, single-institution, observational study we collected clinical data from mCRC patients who received aflibercept in combination with FOLFIRI in routine clinical practice to describe feasibility and efficacy of this regimen in a real-world population. Aflibercept-FOLFIRI is a feasible second-line treatment for $\mathrm{MCRC}$ in a real-life setting, and PFS in first-line therapy $>12$ months resulted as the only predictive marker of better survival.

Abstract: The addition of aflibercept to FOLFIRI has been demonstrated to improve survival in patients with metastatic colorectal cancer (mCRC) who progressed after receiving a standard oxaliplatinbased regimen. In this retrospective, single-institution, observational study we collected clinical data from mCRC patients who received aflibercept in combination with FOLFIRI in routine clinical practice from October 2012 to March 2021 to describe feasibility and efficacy of this regimen in a real-world population. Forty-nine patients receiving aflibercept-FOLFIRI as second-line treatment were identified, $40.8 \%$ of whom were aged over 65 years. The majority of patients had multi-organ metastases $(73.5 \%)$, and had previously received bevacizumab in combination with chemotherapy (CT) as first-line treatment (79.6\%). Median overall survival (OS) and progression-free survival (PFS) were 13 and 6 months, respectively; overall response rate (ORR) and disease control rate (DCR) were $12.3 \%$ and $49.1 \%$, respectively. Several factors were associated with survival in univariate analysis, including PFS in first-line therapy, number of metastatic sites, bone metastases and others. However, in multivariate analysis, only PFS in first-line CT over 12 months was significantly associated with better OS (HR 0.32; 95\% CI 0.13-0.79; $p=0.01$ ). Hypertension was the most commonly reported grade (G) 3-4 adverse event (AE), affecting $18.4 \%$ of the overall population. Thromboembolic events were observed in $16.3 \%$ of patients, hemorrhagic events in $10.2 \%$, and proteinuria in $8.2 \%$. Neutropenia was the most frequently observed hematological G3-4 AE with an incidence of $10.2 \%$. AfliberceptFOLFIRI has been confirmed as a feasible second-line treatment for mCRC in a re-al-life setting, and PFS in first-line therapy $>12$ months resulted as the only predictive marker of better survival.

Keywords: aflibercept; colorectal cancer; safety; prognostic factors; bone metastasis 


\section{Introduction}

Colorectal cancer (CRC) is the third most commonly diagnosed cancer worldwide, with approximately 1.9 million new cases and 935,000 deaths annually [1].

Since angiogenesis was identified as a fundamental process for tumor development and growth, from the early pathogenesis to clinically evident disease, it was included among the hallmarks of cancer. Angiogenesis is a complex process by which new blood vessels are formed from endothelial precursor, mediated through several ligands and receptors that work in tight regulation [2]. A group of glycoproteins, including the the vascular endothelial growth factors (VEGFs) (VEGF-A, VEGF-B, VEGF-C, and VEGF-D) and the placental growth factor (PIGF), act as effectors of angiogenesis interacting with VEGF receptors and neuropilin co-receptors [3]. In addition, there are multiple isoforms of VEGF-A $\left(\mathrm{VEGF}_{121}, \mathrm{VEGF}_{165}, \mathrm{VEGF}_{189}\right.$, and $\left.\mathrm{VEGF}_{206}\right)$. A crosstalk between the VEGF signaling pathway and other angiogenic signaling pathways such as the angiopoietins (Ang-1, Ang-2, and Ang-4) and the Notch receptors (Notch 1 and Notch 4) and their ligands occurs. Furthermore, integrins and hypoxia (through HIF- $1 \alpha$ and HIF-2 $\alpha$ ) may affect VEGF and other signaling components in tumor angiogenesis [4]. Antiangiogenic therapy constitutes a cornerstone of the treatment of patients with metastatic CRC (mCRC) [5-7]. In the last three decades, monoclonal antibodies (moAbs) against the VEGF/VEGF receptor (VEGFR) pathway and anti-angiogenic multikinase inhibitors have been widely used in the treatment of $\mathrm{mCRC}$, both in the first and subsequent lines of therapy [5-12].

Aflibercept is a fusion protein that binds VEGFA, VEGFB, and PIGF. Acting as a soluble receptor with a higher affinity than the endogenous receptors, it prevents the activation of the angiogenesis [12].

In August 2012, the US Food and Drug Administration (FDA) approved aflibercept in combination with FOLFIRI for the treatment of patients with mCRC who progressed after receiving a standard oxaliplatin-based regimen. The approval was based on the results of the randomized phase III VELOUR trial which showed the superiority of the combination of aflibercept with FOLFIRI over placebo with FOLFIRI in terms of overall survival (OS) (mOS 13.5 vs. 12.1 months, respectively; HR 0.817; 95.34\% CI, 0.713-0.937, $p=0.0032$ ), progression-free survival (PFS) (mPFS 6.9 vs. 4.7 months, respectively; HR $0.758 ; 95 \%$ CI, $0.661-0.869, p<0.0001)$, and overall response rate (ORR) (ORR 19.8\% (95\% CI, $16.4-23.2 \%)$ vs. $11.1 \%(95 \% \mathrm{CI}, 8.5-13.8 \%)$, respectively; $p=0.0001)$. As expected, patients treated with aflibercept reported a higher rate of grade (G) 3-4 adverse events (AEs) (83.5\%) compared to those treated with placebo (62.5\%). In particular, G3-4 hypertension was observed in $19.3 \%$ vs. $1.5 \%$, diarrhea in $19.3 \%$ vs. $7.8 \%$, neutropenia in $36.7 \%$ vs. $29.5 \%$ hemorrhage in $2.9 \%$ vs. $1.7 \%$, arterial thromboembolic events in $1.8 \%$ vs. $0.5 \%$, and venous thromboembolic events in $7.9 \%$ vs. $6.3 \%$ [12].

To date, efficacy and safety of aflibercept plus FOLFIRI in routine clinical practice have been explored in several observational studies and early access programs (Table 1). Overall, the safety profile and real-world treatment feasibility (i.e., incidence of AEs, treatment exposure, anti-VEGF class events, etc.) may differ from the pivotal trials and, as a consequence, the achievement of clinical outcomes may not be optimal.

The purpose of this observational retrospective study was to evaluate the feasibility of aflibercept plus FOLFIRI in a real-world, unselected population, with a series of unfavorable clinical prognostic factors, potentially underrepresented in clinical trials. As additional analysis, this study explored the role of prognostic markers to identify potential subgroups of patients characterized by longer survival. 
Table 1. Summary of the main observational studies and early access programs of aflibercept in mCRC.

\begin{tabular}{|c|c|c|c|c|c|c|c|c|c|c|c|c|c|c|}
\hline Ref. & $\begin{array}{c}\text { Treatment } \\
\text { Setting }\end{array}$ & $\begin{array}{c}\text { Patients } \\
\text { Previously } \\
\text { Treated } \\
\text { with Beva- } \\
\text { cizumab }\end{array}$ & $\begin{array}{l}\text { N. Pa- } \\
\text { tients }\end{array}$ & Known Mutations & $\begin{array}{c}\text { Prognostic } \\
\text { Factors }\end{array}$ & ORR & DCR & $\begin{array}{l}\text { PFS } \\
\text { (Months) }\end{array}$ & $\begin{array}{c}\text { OS } \\
\text { (Months) }\end{array}$ & $\begin{array}{l}\text { G3-4 Hy- } \\
\text { pertension } \\
(\%)\end{array}$ & $\begin{array}{l}\text { Thromboem- } \\
\text { bolic Events } \\
\text { (\%) }\end{array}$ & $\begin{array}{l}\text { Proteinuria } \\
\text { G3-4 (\%) }\end{array}$ & $\begin{array}{c}\text { Dose } \\
\text { Reduction } \\
\text { Rate for } \\
\text { Aflibercept } \\
\text { Due to AEs } \\
\text { (\%) }\end{array}$ & $\begin{array}{l}\text { Discontinuation } \\
\text { Rate for } \\
\text { Aflibercept Due } \\
\text { to AEs (\%) }\end{array}$ \\
\hline $\begin{array}{l}\text { Riechelmann } \\
\text { et al. [12] }\end{array}$ & $\begin{array}{c}\text { In combination } \\
\text { with FOLFIRI } \\
\text { after at least one } \\
\text { prior } \\
\text { oxaliplatin-based } \\
\text { regimen. }\end{array}$ & $46.2 \%$ & 798 & - $\quad$ NA & NA & NA & NA & NA & NA & $24.5 \%$ & $8.6 \%$ & $3.6 \%$ & $19 \%$ & $11.7 \%$ \\
\hline $\begin{array}{c}\text { Chau et al. } \\
\text { [13] }\end{array}$ & $\begin{array}{l}\text { In combination } \\
\text { with FOLFIRI as } \\
\text { second or further } \\
\text { line of treatment. }\end{array}$ & $58.6 \%$ & 766 & $\begin{array}{ll}- & 51.5 \% \\
& \text { KRAS-mutated } \\
\text { CRC } & 35.3 \% \text { KRAS WT } \\
\text { - } & \text { CRC } \\
\bullet & 23.2 \% \text { KRAS NA } \\
- & \text { CRC BRAF-mutated } \\
- & 22.5 \% \text { BRAF WT } \\
\text { - } & \text { CRC } \\
& 74.8 \% \text { BRAF NA }\end{array}$ & None & $16.3 \%$ & $50.8 \%$ & 6.1 & 12.5 & $10.2 \%$ & NA & $2.7 \%$ & $36.2 \%$ & NA \\
\hline $\begin{array}{l}\text { Ivanova } \\
\text { et al. [14] }\end{array}$ & $\begin{array}{c}\text { In combination } \\
\text { with FOLFIRI } \\
\text { after at least one } \\
\text { prior } \\
\text { oxaliplatin-based } \\
\text { regimen. }\end{array}$ & $91.7 \%$ & 218 & $\begin{array}{ll}- & 43.7 \% \text { RAS-mutated } \\
\text { : } & \text { CRC } \\
& 17.9 \% \text { RAS WT WR CRC } \\
\bullet & 3.6 \% \text { BRAF-mutated } \\
\text { - } & \text { CRC } \\
& 17.3 \% \text { BRAF WT } \\
\text { - } & \text { CRC } \\
& 79.1 \% \text { BRAF NA }\end{array}$ & NA & $21.1 \%$ & $38.5 \%$ & 3.7 & 9.6 & $6.4 \%$ & $2.3 \%$ & NA & $9.2 \%$ & $23.4 \%$ \\
\hline $\begin{array}{l}\text { Buchler et al. } \\
{[15]}\end{array}$ & $\begin{array}{l}\text { Second-line } \\
\text { treatment mainly } \\
\text { in combination } \\
\text { with FOLFIRI } \\
\text { after standard } \\
\text { CT plus } \\
\text { bevacizumab. }\end{array}$ & $100 \%$ & 366 & $\begin{array}{ll}\text { - } & \text { 80.8\% RAS-mutated } \\
\text { - } & 12 \mathrm{R} \text { RAS WT CRC } \\
& 6.8 \% \text { RAS NA } \\
\bullet & 5.5 \% \text { BRAF-mutated } \\
\text { - } & \text { CRC } \\
& 17.5 \% \text { BRAF WT } \\
\text { - } & \text { CRC } \\
& 77.0 \% \text { BRAF NA }\end{array}$ & $\begin{array}{l}\text { PFS: } \\
\text { duration of } \\
\text { prior beva- } \\
\text { cizumab } \\
\text { therapy. } \\
\text { OS: } \\
\text { presence of } \\
\text { metastases } \\
\text { at diagnosis. }\end{array}$ & NA & NA & 5.6 & 14.2 & NA & NA & NA & NA & $2.3 \%$ \\
\hline $\begin{array}{c}\text { Vera et al. } \\
{[16]}\end{array}$ & $\begin{array}{l}\text { Second-line } \\
\text { treatment in } \\
\text { combination } \\
\text { with FOLFIRI } \\
\text { after standard } \\
\text { CT plus } \\
\text { anti-EGFR } \\
\text { moAb. }\end{array}$ & $0 \%$ & 120 & $\begin{array}{ll}- & 95 \% \text { RAS/RAF WT } \\
\text { - } & \text { CRC } \\
& 5 \% \text { BRAF-mutated } \\
& \text { CRC }\end{array}$ & $\begin{array}{l}\text { OS: ECOG } \\
\text { PS and } \\
\text { number of } \\
\text { metastatic } \\
\text { sites. }\end{array}$ & $33 \%$ & NA & 6.9 & 14.5 & $7.5 \%$ & NA & NA & $18.3 \%$ & $6.7 \%$ \\
\hline
\end{tabular}


Table 1. Cont.

\begin{tabular}{|c|c|c|c|c|c|c|c|c|c|c|c|c|c|c|}
\hline $\begin{array}{l}\text { Feliu et al. } \\
\text { [17] }\end{array}$ & $\begin{array}{l}\text { Second-line } \\
\text { treatment in } \\
\text { combination } \\
\text { with FOLFIRI. }\end{array}$ & $60.6 \%$ & 71 & $\begin{array}{ll}-\quad & 67.7 \% \\
& \text { KRAS-mutated } \\
\text { CRC } \\
\text { - } 32.4 \% \text { KRAS WT } \\
\text { CRC }\end{array}$ & $\begin{array}{l}\text { PFS: LDH, } \\
\text { ECOG PS. }\end{array}$ & $19.7 \%$ & $57.7 \%$ & 5.3 & NA & $11.3 \%$ & $2.8 \%$ & $1.4 \%$ & $14.1 \%$ & $9.9 \%$ \\
\hline $\begin{array}{c}\text { Yusof et al. } \\
{[18]}\end{array}$ & $\begin{array}{l}\text { Second-line } \\
\text { treatment in } \\
\text { combination } \\
\text { with FOLFIRI. }\end{array}$ & $8 \%$ & 25 & $\begin{array}{ll}- & 20.0 \% \\
& \text { KRAS-mutated } \\
\text { CRC } & 32.0 \% \text { KRAS WT } \\
& \text { CRC } \\
\text { - } & 48.0 \% \text { KRAS NA }\end{array}$ & NA & $25 \%$ & $62.5 \%$ & 6.1 & 12.0 & $8 \%$ & NA & $4 \%$ & NA & NA \\
\hline $\begin{array}{l}\text { Montes et al. } \\
{[19]}\end{array}$ & $\begin{array}{l}\text { Second-line } \\
\text { treatment in } \\
\text { combination } \\
\text { with FOLFIRI. }\end{array}$ & $47.4 \%$ & 78 & $\begin{array}{ll} & 75.7 \% \text { RAS-mutated } \\
& \text { CRC }\end{array}$ & $\begin{array}{l}\text { PFS and OS: } \\
\text { metachronous } \\
\text { metastases, } \\
\text { left-side } \\
\text { primary } \\
\text { tumor } \\
\text { location. }\end{array}$ & 21.8 & $70.5 \%$ & 6.8 & 12.0 & $3.8 \%$ & NA & $0 \%$ & NA & NA \\
\hline $\begin{array}{c}\text { Kim et al. } \\
{[20]}\end{array}$ & $\begin{array}{l}\text { Second-line } \\
\text { treatment in } \\
\text { combination } \\
\text { with FOLFIRI. }\end{array}$ & $96.2 \%$ & 52 & $\begin{array}{ll}\text { - } & 57.7 \% \\
& \text { RAS/RAF-mutated } \\
\text { CRC } & \text { 36. } \% \text { RAS/RAF } \\
\text { - } & \text { WT CRC } \\
& 5.8 \% \text { NA }\end{array}$ & $\begin{array}{c}\text { PFS: } \\
\text { response to } \\
\text { first-line } \\
\text { treatment, } \\
\text { left-side } \\
\text { primary } \\
\text { tumor } \\
\text { location, } \\
\text { low baseline } \\
\text { CEA level, } \\
\text { WT } \\
\text { RAS/RAF. } \\
\text { OS: primary } \\
\text { tumor } \\
\text { location, } \\
\text { baseline } \\
\text { CEA level, } \\
\text { RAS/RAF } \\
\text { mutational } \\
\text { status. }\end{array}$ & $48.1 \%$ & $84.6 \%$ & 7.0 & 16.8 & NA & NA & NA & NA & NA \\
\hline $\begin{array}{l}\text { Chong et al. } \\
\text { [21] }\end{array}$ & $\begin{array}{l}\text { Second-line } \\
\text { treatment in } \\
\text { combination } \\
\text { with FOLFIRI. }\end{array}$ & $15.8 \%$ & 19 & $\begin{array}{ll}- & 42.1 \% \text { RAS-mutated } \\
\text { - } & \text { CRC } \\
- & 52.6 \% \text { RAS WT CRC } \\
- & 5.3 \% \text { RAS NA } \\
\text { - } & \text { CRC } \\
- & 42.1 \% \text { BRAF-mutated } \\
\text { - } & \text { CRC } \mathrm{BRT} \\
- & 52.6 \% \text { BRAF NA }\end{array}$ & NA & $21.1 \%$ & $42.1 \%$ & 4.1 & 11.6 & $5.3 \%$ & $5.3 \%$ & NA & NA & NA \\
\hline
\end{tabular}


Table 1. Cont.

\begin{tabular}{|c|c|c|c|c|c|c|c|c|c|c|c|c|c|c|}
\hline $\begin{array}{l}\text { Ottaiano } \\
\text { et al } \\
\text { (ARM B) } \\
\text { [22] }\end{array}$ & $\begin{array}{l}\text { Second-line } \\
\text { treatment in } \\
\text { combination } \\
\text { with FOLFIRI. }\end{array}$ & $100 \%$ & 43 & $\begin{array}{ll}-\quad & 100 \% \\
\text { KRAS-mutated } \\
\text { CRC }\end{array}$ & NA & $11.6 \%$ & $48.8 \%$ & NA & 12.1 & $4.6 \%$ & $0 \%$ & NA & $2.3 \%$ & NA \\
\hline $\begin{array}{c}\text { Auvray et al. } \\
{[23]}\end{array}$ & $\begin{array}{l}\text { In combination } \\
\text { with CT beyond } \\
\text { second-line } \\
\text { therapy. }\end{array}$ & $86.2 \%$ & 130 & 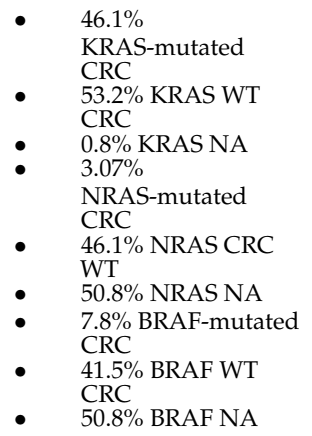 & $\begin{array}{l}\text { PFS and OS: } \\
\text { prior antian- } \\
\text { giogenic } \\
\text { treatment. }\end{array}$ & $6.9 \%$ & $45.4 \%$ & 3.3 & 7.6 & $0.8 \%$ & $0 \%$ & $1.5 \%$ & NA & $1.5 \%$ \\
\hline
\end{tabular}

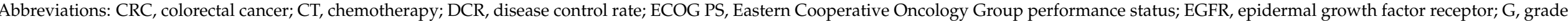
moAbs, monoclonal antibodies; N., number; NA, not assessed; ORR, overall response rate; OS, overall survival; PFS, progression-free survival; WT, wild-type. 


\section{Patients and Methods}

\subsection{Study Population}

In this single-institution, retrospective, observational study, we collected clinical data from patients who received aflibercept in combination with FOLFIRI in routine clinical practice at Clinical Oncology Unit, Careggi University Hospital (Florence, Italy), from October 2012 to March 2021.

The study population consisted of patients with histologically confirmed diagnosis of mCRC who progressed after a first-line oxaliplatin-based chemotherapy (CT). Other inclusion criteria were: age $\geq 18$ years and written informed consent. Patients who received aflibercept for other indications were excluded. The study was conducted in accordance with the Declaration of Helsinki. The protocol was approved by the Comitato Etico Regionale for clinical experimentation of Toscana region (Italy), Area Vasta Centro section, number: 17332_oss.

\subsection{Treatment}

All patients received at least one cycle of aflibercept-FOLFIRI regimen, which consisted of aflibercept $4 \mathrm{mg} / \mathrm{kg}$ on day 1 , irinotecan $180 \mathrm{mg} / \mathrm{m}^{2}$ on day 1 , levofolinate $200 \mathrm{mg} / \mathrm{m}^{2}$ on day 1 , and 5-fluorouracil $400 \mathrm{mg} / \mathrm{m}^{2}$ bolus injection followed by $48 \mathrm{~h}$ continuous infusion of 5 -fluorouracil $2400 \mathrm{mg} / \mathrm{m}^{2}$ on day 1 . All drugs were administered intravenously every two weeks. Given the observational nature of the study, dose modifications, delays, treatment discontinuations, and premedications were performed as per clinical practice.

\subsection{Data Collection and Study Endpoints}

Clinical data including all available demographic information, medical history, diagnosis, treatment, pathological results, molecular analyses (including RAS, BRAF and microsatellite instability (MSI) status), clinical outcomes, AEs, and laboratory alterations were collected from patients' medical records. AEs were graded according to the National Cancer Institute Common Terminology Criteria for AEs version 4.03 [24] Tumor response evaluation was performed at least every 3 months, or before if clinically indicated by a chest-abdomen computed tomography. Radiological response was assessed according to RECIST, version 1.1 [25]. Disease progression was assessed as either radiological or clinical progression.

The primary endpoint was the evaluation of the efficacy in terms of OS. Secondary endpoints included PFS, ORR, disease control rate (DCR), safety, and the evaluation of treatment adherence and potential prognostic factors.

OS was defined as the time from the start of treatment to death from any cause. PFS was defined as the time from the beginning of the treatment to disease progression or death from any cause.

\subsection{Statistical Analysis}

Treatment adherence, safety, and correlation of clinical, biological, pathological factors and survival outcomes were analyzed. Statistical comparisons for categorical variables were performed using $\chi^{2}$ test. Time-to-event endpoints were estimated using the KaplanMeier method. Survival distributions for specific subgroups of patients were tested with a log-rank test. A $p$-value of 0.05 or lower was considered to be statistically significant. Parameters with a statistically significant log-rank test were considered independent variables and included in the multivariate Cox proportional hazard regression linear model to compare hazard ratio (HR) and 95\% confidence interval (95\% CI). All analyses were performed using the STATA, version 12.0; StataCorp LLC, College Station TX, USA. 


\section{Results}

\subsection{Patient Characteristics}

From October 2012 to March 2021, 49 patients with mCRC treated with afliberceptFOLFIRI as second-line treatment were identified. Patients' characteristics are described in Table 2. Twenty-seven patients (55.1\%) were female and 22 (44.9\%) were male; $40.8 \%$ of patients $(n=20)$ were aged over 65 years. Eastern Cooperative Oncology Group performance status (ECOG PS) was 0 in $77.6 \%$ of patients and 1 in $22.4 \%$. The prevalent primary tumor location was left $(67.3 \%) ; 18$ patients $(36.7 \%)$ had a mucinous component at the histological evaluation. Thirty-six patients (73.5\%) had multi-organ metastases, including liver (73.5\%), lung (51\%), lymph nodes (49\%), and peritoneum (26.5\%). KRAS mutation was detected in 34 patients $(69.4 \%)$ and NRAS mutation in three $(6.1 \%)$; BRAF mutation was found in two out of 41 samples analyzed (4.9\%). Among patients with MSI data available $(n=18), 11.1 \%$ had MSI-high tumor. The majority of patients $(89.8 \%)$ had a weight loss of more than five kilograms in the last three months.

Table 2. Characteristics of patients.

\begin{tabular}{|c|c|}
\hline Baseline Characteristics & N. of Patients $(n=49)$ \\
\hline \multicolumn{2}{|l|}{ Sex: } \\
\hline Male & $22(44.9 \%)$ \\
\hline Female & $27(55.1 \%)$ \\
\hline \multicolumn{2}{|l|}{ Age: } \\
\hline$\geq 65$ & $20(40.8 \%)$ \\
\hline$<65$ & $29(59.2 \%)$ \\
\hline \multicolumn{2}{|l|}{ ECOG PS: } \\
\hline 0 & $38(77.6 \%)$ \\
\hline 1 & $11(22.4 \%)$ \\
\hline \multicolumn{2}{|l|}{ Primary tumor location: } \\
\hline Left & $33(67.3 \%)$ \\
\hline Right & $16(32.7 \%)$ \\
\hline \multicolumn{2}{|l|}{ N. of metastatic organs involved at baseline: } \\
\hline 1 & $13(26.5 \%)$ \\
\hline$>1$ & $36(73.5 \%)$ \\
\hline \multicolumn{2}{|l|}{ Metastatic organs involved at baseline: } \\
\hline Liver & $36(73.5 \%)$ \\
\hline Peritoneum & $13(26.5 \%)$ \\
\hline Bone & $3(6.1 \%)$ \\
\hline Lymph nodes & $24(49.0 \%)$ \\
\hline Lung & $25(51.0 \%)$ \\
\hline \multicolumn{2}{|l|}{ Mucinous component: } \\
\hline Yes & $18(36.7 \%)$ \\
\hline No & $31(63.3 \%)$ \\
\hline \multicolumn{2}{|l|}{ RAS, RAF, MSI status: } \\
\hline KRAS-mutated & $34(69.4 \%)$ \\
\hline NRAS-mutated & $3(6.1 \%)$ \\
\hline BRAF-mutated among patients tested $(n=41)$ & 2 out of $41(4.9 \%)$ \\
\hline MSI-H among patients tested $(n=18)$ & 2 out of $18(11.1 \%)$ \\
\hline \multicolumn{2}{|l|}{ Weight loss in last 3 months: } \\
\hline$\geq 5 \mathrm{~kg}$ & $44(89.8 \%)$ \\
\hline$<5 \mathrm{~kg}$ & $5(10.2 \%)$ \\
\hline
\end{tabular}

Abbreviations: CRC, colorectal cancer; ECOG PS, Eastern Cooperative Oncology Group performance status; MSI microsatellite instability; N, number.

Thirty-four patients (69.4\%) had received previous surgery of the primary tumor and nine $(18.4 \%)$ had received adjuvant CT. The most frequent best response to first-line CT was partial response (PR) in $40.8 \%$ and stable disease (SD) in $24.5 \%$, with 6-month PFS and 
12-month PFS rates of $77.6 \%$ and $35.9 \%$, respectively. The majority of patients $(79.6 \%)$ had received bevacizumab in combination with $\mathrm{CT}$ as first-line treatment (Table 3 ).

Table 3. Characteristics of patients concerning prior therapies.

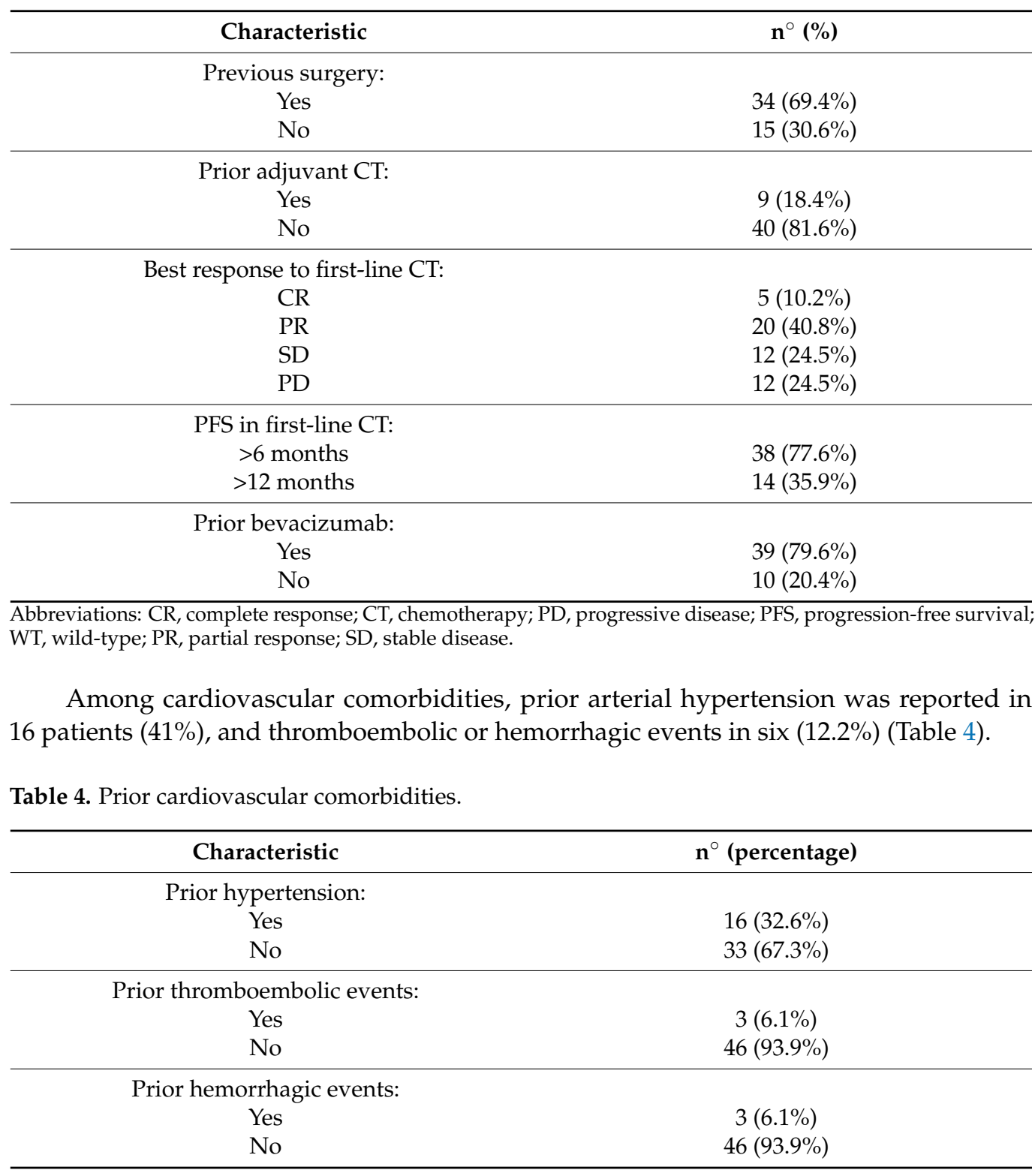

\subsection{Treatment}

During the study, patients received a median of seven cycles of treatment (range 1-13) and 17 patients (43.7\%) more than 10 cycles. CT and aflibercept dose reduction occurred in $35(71.4 \%)$ and $13(26.5 \%)$ patients, respectively. Twenty patients $(40.8 \%)$ required treatment interruption (FOLFIRI, aflibercept or both) due to AEs. Thirty-one patients (63.3\%) received further lines of treatment: trifluridine/tipiracil $(n=10)$, regorafenib $(n=10)$, and other regimens in 11 patients (Table 5). 
Table 5. Best response, PFS, OS, and treatment data.

\begin{tabular}{|c|c|}
\hline Characteristic & $\begin{array}{l}\text { All Patients } \\
(n=49) \\
\mathrm{N}^{\circ}(\%)\end{array}$ \\
\hline CR & $2(4.1 \%)$ \\
\hline PR & $4(8.2 \%)$ \\
\hline SD & $18(36.7 \%)$ \\
\hline PD & $22(44.9 \%)$ \\
\hline ORR & $6(12.3 \%)$ \\
\hline DCR & $24(49.1 \%)$ \\
\hline NA & $3(6.1 \%)$ \\
\hline PFS M-months $(95 \% \mathrm{CI})$ & $6(5-7)$ \\
\hline OS M-months (95\% CI) & $13(10-18)$ \\
\hline $\begin{array}{l}\text { Cycles } \\
\text { Median } \\
\text { Range } \\
>10\end{array}$ & $\begin{array}{c}\quad 7 \\
1-13 \\
17(43.7 \%)\end{array}$ \\
\hline Dose reduction & $\begin{array}{c}\text { CT: } 35(71.4 \%) \\
\text { Aflibercept: } 13(26.5 \%)\end{array}$ \\
\hline Treatment Interruption (aflibercept, FOLFIRI or both) due to AEs & $20(40.8 \%)$ \\
\hline $\begin{array}{c}\text { Further Lines of treatment } \\
\text { TAS-102 } \\
\text { Regorafenib } \\
\text { Other }\end{array}$ & $\begin{array}{c}31(63.3 \%) \\
10 \\
10 \\
11\end{array}$ \\
\hline
\end{tabular}

Abbreviations: $\mathrm{AE}$, adverse event; $\mathrm{CR}$, complete response; $\mathrm{CT}$, chemotherapy; DCR, disease control rate; $\mathrm{ECOG}$ PS, Eastern Cooperative Oncology Group performance status; M, median; N, number; NA, not assessed; ORR, overall response rate; $\mathrm{OS}$, overall survival; $\mathrm{PD}$, progressive disease; $\mathrm{PR}$, partial response; $\mathrm{PFS}$, progression-free survival; SD, stable disease.

\subsection{Efficacy}

Overall, the median OS was 13 months (95\% CI; 10-18) and median PFS was 6 months (95\% CI 5-7) (Figure 1). Complete response (CR), PR, and SD were recorded in $2(4.1 \%), 4$ $(8.2 \%)$ and $18(36.7 \%)$ patients, respectively, yielding an ORR and DCR of $12.3 \%$ and $49.1 \%$, respectively. Response was not evaluable for three patients (6.1\%) (Table 5).

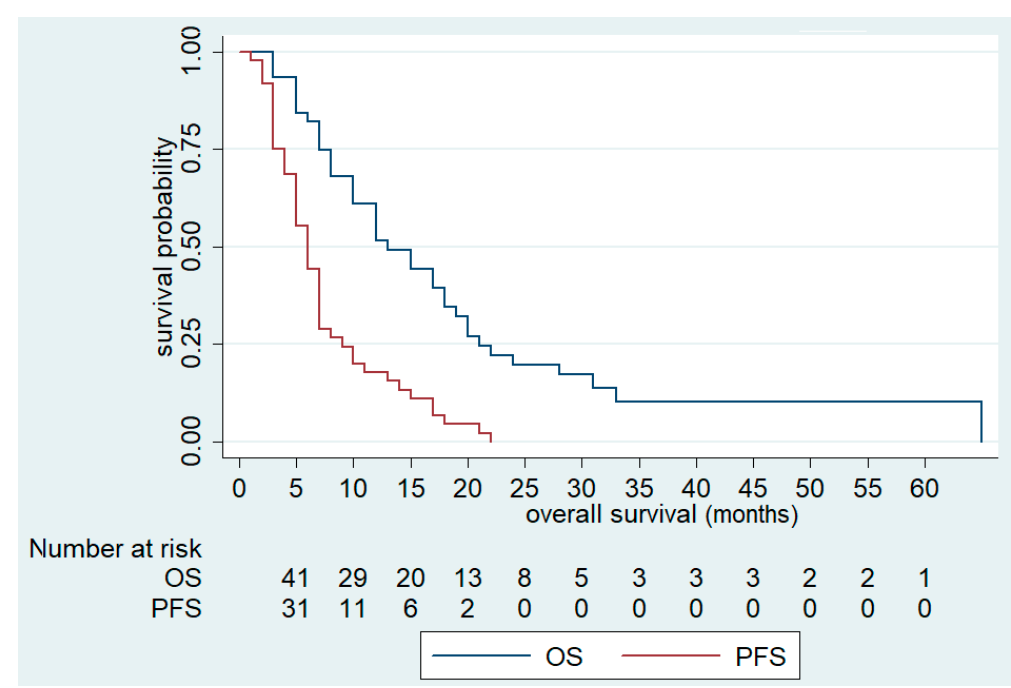

Figure 1. Overall Survival (OS) and Progression-Free Survival (PFS) of FOLFIRI+aflibercept in the overall population. 
Univariate analysis showed that ECOG PS 1, number of metastatic sites $>1$, liver metastases, and bone metastases were negatively associated with OS. In fact, OS was significantly reduced in patients with ECOG PS 1 (10 (95\% CI; 3-12) vs. 17 months (95\% CI $10-20), p=0.03)$, more than one metastatic site involved (12 (95\% CI 8-17) vs. 21 months (95\% CI 12-not reached), $p=0.03)$, liver metastases (12 (95\% CI 7-18) vs. 21 months (95\% CI 12-not reached), $p=0.03$ ), and bone metastases (3 (95\% CI 3-not reached) vs. 15 months (95\% CI 10-19), $p<0.01$ ) (Table 6, Figures 2, S1 and S2).

Table 6. Univariate and multivariate analysis for OS (N. of patients $=49$ ).

\begin{tabular}{|c|c|c|c|c|c|c|}
\hline \multirow[t]{2}{*}{ Characteristic } & \multicolumn{3}{|c|}{ Univariate } & \multicolumn{3}{|c|}{ Multivariate } \\
\hline & HR & CI $95 \%$ & $p$ & HR & CI 95\% & $p$ \\
\hline ECOG PS (1 vs. 0) & 2.31 & $1.01-5.28$ & 0.03 & 1.32 & $0.47-3.74$ & 0.6 \\
\hline Sex (male vs. female) & 1.30 & $0.68-2.48$ & 0.4 & & & \\
\hline Primary site (right vs. left) & 1.43 & $0.71-2.89$ & 0.3 & & & \\
\hline N. of metastatic sites $>1$ & 2.27 & $1.03-5.03$ & 0.03 & 2.18 & $0.67-7.03$ & 0.2 \\
\hline Liver metastases (yes vs. no) & 2.31 & $1.01-5.28$ & 0.03 & 0.79 & $0.23-2.73$ & 0.7 \\
\hline Bone metastases (yes vs. no) & 7.00 & $1.50-32.66$ & $<0.01$ & 3.52 & $0.64-19.31$ & 0.1 \\
\hline Lung metastases (yes vs. no) & 1.60 & $0.80-3.20$ & 0.2 & & & \\
\hline KRAS WT (yes vs. no) & 0.85 & $0.40-1.80$ & 0.7 & & & \\
\hline NRAS WT (yes vs. no) & 1.39 & $0.33-5.81$ & 0.6 & & & \\
\hline BRAF WT (yes vs. no) & 0.47 & $0.10-2.05$ & 0.3 & & & \\
\hline $\begin{array}{l}\text { Mucinous component } \\
\text { (yes vs. no) }\end{array}$ & 0.87 & $0.44-1.74$ & 0.7 & & & \\
\hline $\begin{array}{l}\text { Weight loss in last } 3 \text { months } \\
\text { (no vs. yes) }\end{array}$ & 1.15 & $0.41-3.28$ & 0.7 & & & \\
\hline $\begin{array}{l}\text { Prior bevacizumab (yes vs. } \\
\text { no) }\end{array}$ & 1.25 & $0.55-2.86$ & 0.5 & & & \\
\hline $\begin{array}{l}\text { Prior adjuvant CT } \\
\quad \text { (yes vs. no) }\end{array}$ & 0.57 & $0.22-1.47$ & 0.2 & & & \\
\hline $\begin{array}{l}\text { PFS in first-line CT > } 6 \\
\text { months (yes vs. no) }\end{array}$ & 0.66 & $0.30-1.41$ & 0.3 & & & \\
\hline $\begin{array}{l}\text { PFS in first-line CT > } 12 \\
\text { months (yes vs. no) }\end{array}$ & 0.33 & $0.15-0.72$ & $<0.01$ & 0.32 & $0.13-0.79$ & 0.01 \\
\hline Maintenance (yes vs. no) & 0.44 & $0.21-0.89$ & 0.02 & 0.73 & $0.32-1.65$ & 0.5 \\
\hline Cycles of therapy $>10$ & 0.50 & $0.25-1.00$ & 0.04 & 0.50 & $0.22-1.16$ & 0.1 \\
\hline Age > 65 (yes vs. no) & 1.28 & $0.67-2.45$ & 0.5 & & & \\
\hline $\begin{array}{l}\text { Neutropenia G3-4 } \\
\text { (yes vs. no) }\end{array}$ & 0.84 & $0.29-2.41$ & 0.7 & & & \\
\hline Diarrhea G3-4 (yes vs. no) & 1.95 & $0.72-5.25$ & 0.2 & & & \\
\hline $\begin{array}{l}\text { Hypertension G1/2 } \\
\text { (yes vs. no) }\end{array}$ & 1.20 & $0.61-2.35$ & 0.6 & & & \\
\hline $\begin{array}{l}\text { Hypertension G3-4 } \\
\text { (yes vs. no) }\end{array}$ & 0.65 & $0.28-1.50$ & 0.3 & & & \\
\hline
\end{tabular}

Abbreviations: CT, chemotherapy; ECOG PS, Eastern Cooperative Oncology Group performance status; G, grade $\mathrm{N}$, number; OS, overall survival; PFS, progression-free survival; WT, wild-type.

In contrast, OS was significantly improved in patients who had PFS in first-line CT $>12$ months (28 (95\% CI 13-33) vs. 12 months (95\% CI 7-15), $p<0.01)$, who received maintenance treatment with aflibercept (20 (95\% CI 13-33) vs. 12 months (95\% CI 7-15), 
$p=0.02)$, and number of cycles of aflibercept-FOLFIRI $>10(17$ (95\% CI 12-2)) vs. 12 months (95\% CI 7-18), $p=0.04$ ) (Figures 3 and 4). Other variables, including primary site $(p=0.3)$ and RAS status $(p=0.7)$, did not show statistically significant effects on survival (Table 6).

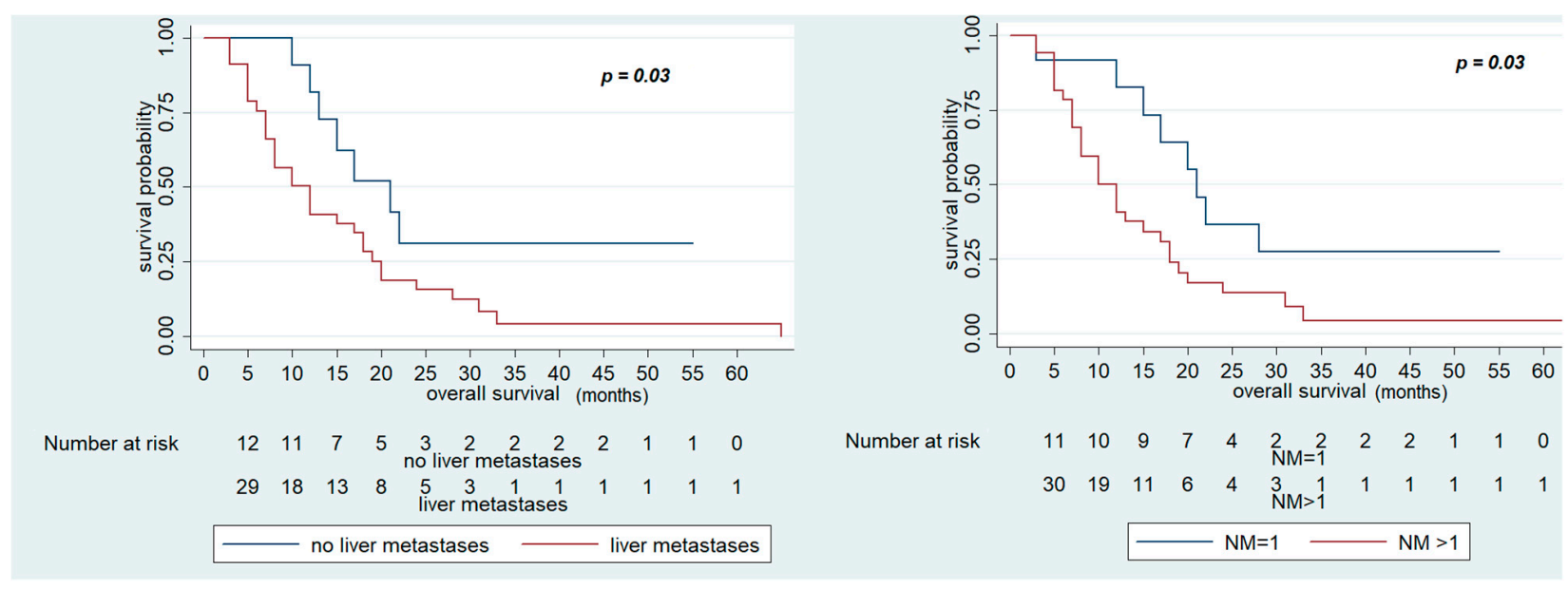

Figure 2. Overall Survival (OS) of FOLFIRI+ aflibercept according to the presence of liver metastases and number of metastatic sites.

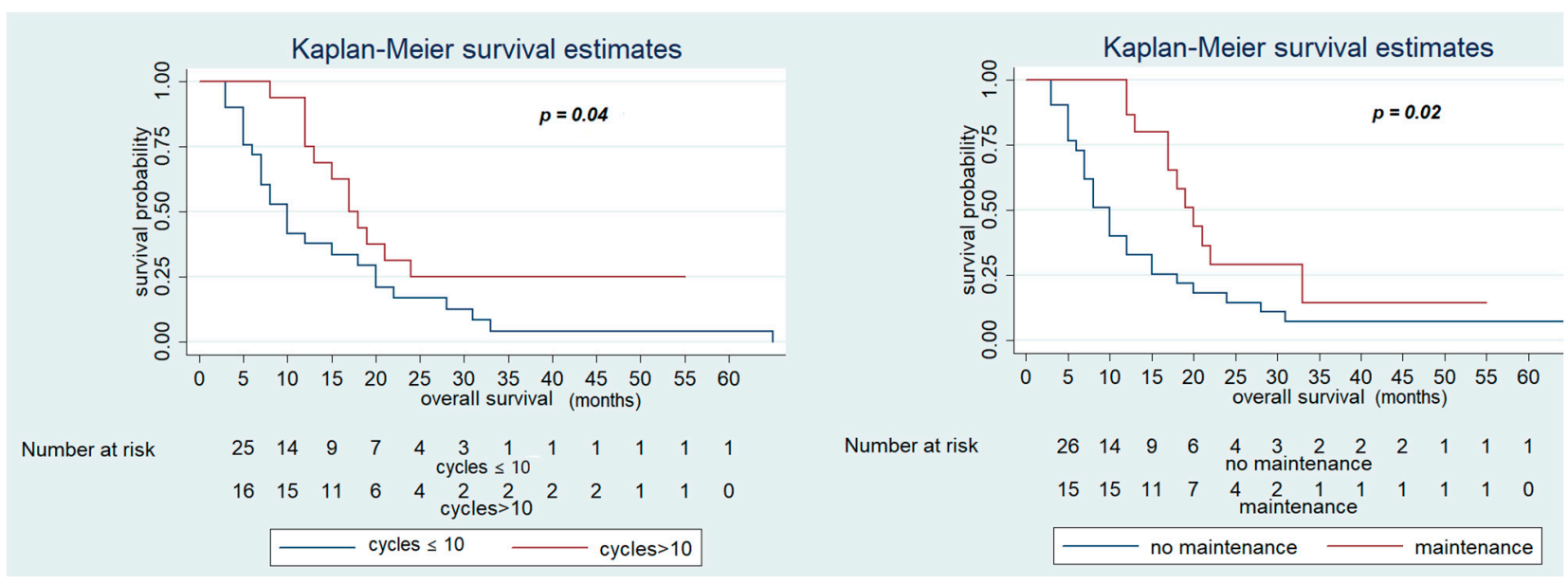

Figure 3. Overall Survival of FOLFIRI+ aflibercept according to the maintenance treatment and number of cycles of therapy $>10$.

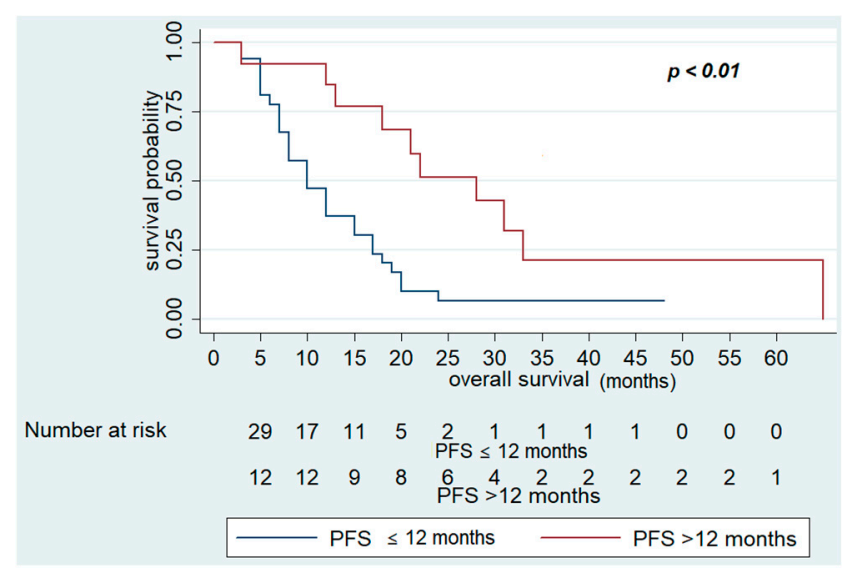

Figure 4. Overall Survival of FOLFIRI+ aflibercept according to PFS in first-line chemotherapy $>12$ months. 
Multivariate analysis confirmed that PFS in first-line CT $>12$ months (HR 0.32; 95\% CI $0.13-0.79 ; p=0.01$ ) was associated with better OS. On the other hand, no variables examined were associated with poor survival in multivariate analysis (Table 6, Figure 4).

As regards PFS, univariate analysis showed that sex (male vs. female) was negatively associated with PFS ( 5 vs. 7 months, $p=0.02$ ). No variables were associated with better PFS in multivariate analysis (Table 7).

Table 7. Univariate analysis for PFS (N. of patients $=49)$.

\begin{tabular}{|c|c|c|c|}
\hline Characteristic & HR & CI $95 \%$ & $p$ \\
\hline ECOG PS (1 vs. 0) & 1.75 & $0.80-3.80$ & 0.1 \\
\hline Sex (male vs. female) & 1.97 & $1.07-3.64$ & 0.02 \\
\hline Site (right vs. left) & 0.99 & $0.52-1.90$ & 0.9 \\
\hline N. of metastatic sites $>1$ & 1.48 & $0.77-2.85$ & 0.2 \\
\hline Liver metastases (yes vs. no) & 1.70 & $0.88-3.28$ & 0.1 \\
\hline Bone metastases (yes vs. no) & 3.73 & $0.81-17.07$ & 0.1 \\
\hline Lung metastases (yes vs. no) & 0.78 & $0.43-1.41$ & 0.4 \\
\hline KRAS WT (yes vs. no) & 1.27 & $0.66-2.44$ & 0.5 \\
\hline NRAS WT (yes vs. no) & 0.47 & $0.14-1.55$ & 0.2 \\
\hline BRAF WT (yes vs. no) & 1.30 & $0.30-5.48$ & 0.7 \\
\hline Mucinous component (yes vs. no) & 0.60 & $0.32-1.14$ & 0.1 \\
\hline Weight loss in last 3 months (no vs. yes) & 0.82 & $0.32-2.12$ & 0.7 \\
\hline Prior bevacizumab (yes vs. no) & 1.44 & $0.71-2.94$ & 0.3 \\
\hline Prior adjuvant CT (yes vs. no) & 0.85 & $0.39-1.33$ & 0.7 \\
\hline PFS in first-line CT > 6 months (yes vs. no) & 0.85 & $0.42-1.73$ & 0.6 \\
\hline PFS in first-line CT > 12 months (yes vs. no) & 0.76 & $0.40-1.48$ & 0.4 \\
\hline Age > 65 (yes vs. no) & 1.42 & $0.76-2,73$ & 0.3 \\
\hline Neutropenia G1-2 (yes vs. no) & 1.15 & $0.57-2.32$ & 0.7 \\
\hline Neutropenia G3-4 (yes vs. no) & 0.90 & $0.32-2.57$ & 0.8 \\
\hline Diarrhea G1/2 (yes vs. no) & 0.54 & $0.29-1.02$ & 0.06 \\
\hline Diarrhea G3/4 (yes vs. no) & 0.97 & $0.38-2.49$ & 0.9 \\
\hline Hypertension G1/2 (yes vs. no) & 0.68 & $0.47-1.26$ & 0.2 \\
\hline Hypertension G3/4 (yes vs. no) & 0.47 & $0.21-1.07$ & 0.07 \\
\hline
\end{tabular}

Abbreviations: CT, chemotherapy; ECOG PS, Eastern Cooperative Oncology Group performance status; G, grade; $\mathrm{N}$, number; PFS, progression-free survival; WT, wild-type.

\subsection{Safety}

Hematological and non-hematological AEs are shown in (Table 8).

Overall, treatment was well tolerated, and most non-hematological toxicities were reported as G1 or 2 (e.g., asthenia in $67.3 \%$ of patients, diarrhea in $55.1 \%$, arterial hypertension in $44.9 \%$, and stomatitis in $34.7 \%$ ). Hypertension was the most commonly reported G3-4 $\mathrm{AE}$, affecting 9 out of 49 patients $(18.4 \%)$. In addition, arterial or venous thromboembolism was observed in eight patients $(16.3 \%)$, hemorrhagic events in five $(10.2 \%)$, fistulas in two $(4.1 \%)$, and proteinuria in four $(8.2 \%)$. No cases of gastrointestinal perforation or severe heart failure were reported.

Neutropenia was the most frequently observed hematological AE with an incidence of $10.2 \%$. Notably, three patients $(6.1 \%)$ had infectious complications.

Maintenance therapy did not significantly increase toxicity since all of the G3-4 AEs occurred during induction therapy. 
Table 8. Most relevant adverse events (AEs) and grade.

\begin{tabular}{|c|c|}
\hline $\mathrm{AE}$ & N. of Patients $(n=49)$ \\
\hline Leukopenia G1/2 & $13(26.5 \%)$ \\
\hline Leukopenia G3/4 & $2(4.1 \%)$ \\
\hline Neutropenia G1/2 & $12(24.5 \%)$ \\
\hline Neutropenia G3/4 & $5(10.2 \%)$ \\
\hline Platelet count decreased G1/2 & $3(6.1 \%)$ \\
\hline Platelet count decreased G3/4 & $1(2.0 \%)$ \\
\hline Diarrhea G1/2 & $27(55.1 \%)$ \\
\hline Diarrhea G3/4 & $5(10.2 \%)$ \\
\hline Proteinuria & $4(8.2 \%)$ \\
\hline Asthenia G1/2 & $33(67.3 \%)$ \\
\hline Asthenia G3 & $7(14.3 \%)$ \\
\hline Arterial hypertension G1/2 & $22(44.9 \%)$ \\
\hline Arterial hypertension G3/4 & $9(18.4 \%)$ \\
\hline Hypertransaminasemia G1/2 & $8(16.3 \%)$ \\
\hline Hypertransaminasemia G3/4 & $1(2.0 \%)$ \\
\hline Stomatitis G1/2 & $17(34.7 \%)$ \\
\hline Stomatitis G3/4 & $3(6.1 \%)$ \\
\hline Infectious complications & $3(6.1 \%)$ \\
\hline Hypersensitivity & $2(4.1 \%)$ \\
\hline Arterial/venous thromboembolism & $8(16.3 \%)$ \\
\hline Perforation & 0 \\
\hline Hemorrhagic events & $5(10.2 \%)$ \\
\hline Fistulas & $2(4.1 \%)$ \\
\hline Heart failure & 0 \\
\hline
\end{tabular}

Abbreviations: AE, adverse event; $\mathrm{G}$, grade; $\mathrm{N}$, number.

\section{Discussion}

The continuum of care for mCRC might include anti-angiogenic drug as anti-VEGF/VEGFR moAb and recombinant proteins in combination with fluoropyrimidine-based regimens in first- and second-line treatment, and multikinase inhibitors in refractory patients. Since the publication of the results of the VELOUR trial, aflibercept in combination with FOLFIRI has been widely used as second-line treatment in patients with mCRC. [12] However, outside the clinical trial framework, a few case series have explored the feasibility of aflibercept therapy in a non-favorably selected population (Table 1). The main evidence came from the Aflibercept Safety and health-related Quality-of-life Program (ASQoP) (number of patients, 798), prospective post-authorization safety OZONE study $(n=766)$, and two retrospective studies conducted by Ivanova et al. $(n=218)$ and Buchler et al. $(n=366)$ [13-15]. Furthermore, the clinical and molecular factors to select patients who are most likely to benefit from this treatment are largely unexplored.

Despite all the limitations of cross-trial comparison, our patient population was slightly older than that in the VELOUR study, with $40.8 \%$ of patients with $\geq 65$ years compared with $36.1 \%$, and was characterized by a higher tumor burden: multi-organ metastases in $73.5 \%$ of patients compared with $56.3 \%$ [26] (Table 2).

Although our patients were less-favorably selected than those enrolled in clinical trials, survival outcomes were consistent with the literature [12] (Tables 1 and 5). In the pivotal trial, the benefit from aflibercept was observed across the prespecified subgroups, 
including patients who were exposed to bevacizumab in first-line treatment, and this subgroup accounted for only $30 \%$ of the overall population [9,13]. In contrast, in large case series, nearly half of patients with mCRC usually receive bevacizumab in combination with CT as first-line treatment (e.g., $46.2 \%$ in ASQoP, $58.6 \%$ in OZONE, etc.) [13,27] (Table 1), and this was in line with our observational data, with $79.6 \%$ of patients previously treated with bevacizumab (Table 3).

In our study, PFS in first-line therapy $>12$ months was the main factor associated with better OS in multivariate analysis (Table 6). In contrast, other factors, such as prior bevacizumab treatment, RAS and RAF mutational status, site of primary tumor, and hypertension, were not associated with survival (Tables 6, S1 and S2). Multi-organ involvement was associated with poor survival in univariate analysis: this was notably true for patients with bone metastases who had a median OS of 3 months (Table 6 and Figure S2). Although the number of patients was relatively small and results were not significant in multivariate analysis, this observation was consistent with other retrospective studies confirming bone metastases as a poor prognostic factor in mCRC. In fact, presence of bone metastases was usually associated with high tumor burden and reduced survival [28,29]. Notably, tumor burden being significantly related with survival was also suggested in the observational studies of Buchler et al. and Vera et al. [15,16].

To date, predictive factors of response to aflibercept are largely unknown. In a subgroup analysis from the VELOUR trial, a significantly greater treatment benefit from aflibercept was observed for patients with liver-only metastases, compared to those with multi-organ metastases or with other organs involved except the liver [26]. In addition, although the benefit from aflibercept was confirmed across all molecular subgroups, in a biomarker analysis from the VELOUR trial, a trend toward a deeper benefit was observed in RAS WT rather than in RAS-mutated CRC, with median OS of 16.0 months in the aflibercept group vs. 11.7 months in the placebo group for RAS WT patients (HR 0.7), and 12.6 vs. 11.2 months for RAS-mutated patients (HR 0.93) [30]. To date, only a retrospective observational study evaluated aflibercept-FOLFIRI as second-line treatment in a homogenous series of 120 WT RAS CRC patients who had received a first-line standard CT plus anti-EGFR (epidermal growth factor receptor) moAb, describing an ORR of $33 \%$, a median PFS of 6.9 months (95\% CI: 6.1-7.8) and a median OS of 14.5 months (95\% CI: 9.7-19.3) [27]. Unfortunately, our study could not address this crucial issue, due to sample limitation.

The safety profile of aflibercept in combination with FOLFIRI, before its marketing authorization, has been extensively studied in the ASQoP conducted by Riechelmann et al. [31] The patient population $(n=798)$ was approximately representative of the routine clinical practice. Nearly half of the patients had received bevacizumab in combination with CT in first-line treatment. Dose modifications and dose interruption of aflibercept due to $\mathrm{AE}$ occurred in $19 \%$ and $11.7 \%$, respectively. Overall, $78 \%$ of patients experienced G3-4 AEs, including hypertension in $24.5 \%$, neutropenia in $24.8 \%$, and proteinuria in $3.6 \%$. Fifteen treatment-related deaths $(1.9 \%)$ were observed. However, a generally favorable patient's perspective was reported in health-related quality of life scores.

As expected, the safety profile of aflibercept in our patient population was consistent with the VELOUR trial [9], ASQoP, and other case series (Table 1). Among the anti-VEGF class events, hypertension was the most commonly reported G3-4 AE both in our study and in VELOUR trial, affecting $18.4 \%$ and $19.3 \%$ of patients, respectively [12] (Table 8). Although cross-trial comparisons are not formally correct and may not provide definite conclusions, numerically lower rates of G3-4 hypertension were reported with the use of bevacizumab or ramucirumab in the TML, BEBYP, and RAISE trials $(2 \%, 2 \%$, and $11 \%$, respectively) compared with those reported with aflibercept in the VELOUR trial and early access programs $[9,12,32,33]$ (Table 1).

Notably, a higher number of potentially treatment-related any-grade thromboembolic events were observed in our case series $(16.3 \%)$ as compared with those reported in the VELOUR trial (11.9\%), ASQoP (8.6\%), and other case series [12] (Tables 1 and 8). These events deserve special attention and early management, as patients may experience 
long-term and potentially serious consequences. As previously reported in the literature, predictors of thromboembolic events in CRC included metastatic disease, number of comorbidities, and, more recently, the use of angiogenesis inhibitors [34,35]. In our study, the high rates of patients previously treated with bevacizumab, with multi-organ metastases and cardiovascular comorbidities might have influenced the incidence of thromboembolic events.

This study had several limitations, including the relatively small sample size, retrospective design, and lack of a control group that precluded a precise estimate of treatment benefit.

\title{
5. Conclusions
}

In conclusion, our study confirmed aflibercept-FOLFIRI as a feasible second-line treatment of $\mathrm{mCRC}$ in a patient population approximately representative of daily clinical practice, with PFS in first-line CT $>12$ months as the main predictor of better survival.

Supplementary Materials: The following are available online at https://www.mdpi.com/article/ $10.3390 /$ cancers13153863/s1. Figure S1. Overall Survival of FOLFIRI + aflibercept according to ECOG, Figure S2. Overall Survival of FOLFIRI + aflibercept according to presence of bone metastases, Table S1. Treatment re-sponse according to KRAS status, Table S2. Treatment response according to primary site.

Author Contributions: Conceptualization, D.L., L.A., and S.P., methodology, G.R. and A.A.; validation, L.D. and M.B.; formal analysis, G.R.; data curation, S.D. and D.L.; writing-original draft preparation, D.L., G.R.; writing — review and editing, S.P., E.G. and L.A.; supervision, L.A. All authors have read and agreed to the published version of the manuscript.

Funding: This research received no external funding.

Institutional Review Board Statement: The study was conducted in accordance with the Declaration of Helsinki. The protocol was approved by the Comitato Etico Regionale for clinical experimentation of Toscana region (Italy), Area Vasta Centro section, number: 17332_oss (19 May 2020).

Informed Consent Statement: Informed consent was obtained from all subjects involved in the study.

Data Availability Statement: Data available on request to the corresponding author.

Conflicts of Interest: The authors declare no conflict of interest.

\begin{abstract}
Abbreviations
AE: adverse event; ASQoP: Aflibercept Safety and health-related Quality-of-life Program; CR: complete response; CRC: colorectal cancer; CT: chemotherapy; DCR: disease control rate; ECOG PS: Eastern Cooperative Oncology Group performance status; EGFR: epidermal growth factor receptor; FDA: Food and Drug Administration; G: grade; mCRC: metastatic colorectal cancer; moAbs: monoclonal antibodies; MSI: microsatellite instability; ORR: overall response rate; OS: overall survival; PFS: progression-free survival; PIGF: placental growth factor; PR: partial response; SD: stable disease; VEGF: vascular endothelial growth factor; VEGFR: vascular endothelial growth factor receptor.
\end{abstract}

\section{References}

1. Araghi, M.; Soerjomataram, I.; Bardot, A.; Ferlay, J.; Cabasag, C.J.; Morrison, D.S.; De, P.; Tervonen, H.; Walsh, P.M.; Bucher, O.; et al. Changes in colorectal cancer incidence in seven high-income countries: A population-based study. Lancet Gastroenterol. Hepatol. 2019, 4, 511-518. [CrossRef]

2. Jain, R.K. Normalization of Tumor Vasculature: An Emerging Concept in Antiangiogenic Therapy. Science 2005, 307, 58-62. [CrossRef]

3. Mousa, L.; Salem, M.E.; Mikhail, S. Biomarkers of Angiogenesis in Colorectal Cancer. Biomark. Cancer 2015, 7, BIC-S25250. [CrossRef]

4. Sun, W. Angiogenesis in metastatic colorectal cancer and the benefits of targeted therapy. J. Hematol. Oncol. 2012, 5, 1-9. [CrossRef] [PubMed]

5. Hanahan, D.; Weinberg, R.A. Hallmarks of Cancer: The Next Generation. Cell 2011, 144, 646-674. [CrossRef] [PubMed] 
6. Raica, M.; Cimpean, A.M.; Ribatti, D. Angiogenesis in pre-malignant conditions. Eur. J. Cancer 2009, 45, 1924-1934. [CrossRef] [PubMed]

7. National Comprehensive Cancer Network. Colon Cancer (Version 2.2021). Available online: https://www.nccn.org/ professionals/physician_gls/pdf/colon.pdf (accessed on 27 June 2021).

8. Cremolini, C.; Loupakis, F.; Antoniotti, C.; Lupi, C.; Sensi, E.; Lonardi, S.; Mezi, S.; Tomasello, G.; Ronzoni, M.; Zaniboni, A.; et al. FOLFOXIRI plus bevacizumab versus FOLFIRI plus bevacizumab as first-line treatment of patients with metastatic colorectal cancer: Updated overall survival and molecular subgroup analyses of the open-label, phase 3 TRIBE study. Lancet Oncol. 2015, 16, 1306-1315. [CrossRef]

9. Tabernero, J.; Yoshino, T.; Cohn, A.L.; Obermannova, R.; Bodoky, G.; Garcia-Carbonero, R.; Ciuleanu, T.E.; Portnoy, D.C.; Van Cutsem, E.; Grothey, A.; et al. Ramucirumab versus placebo in combination with second-line FOLFIRI in patients with metastatic colorectal carcinoma that progressed during or after first-line therapy with bevacizumab, oxaliplatin, and a fluoropyrimidine (RAISE): A randomised, double-blind, multicentre, phase 3 study. Lancet Oncol. 2015, 16, 499-508. [CrossRef]

10. Hurwitz, H.; Fehrenbacher, L.; Novotny, W.; Cartwright, T.; Hainsworth, J.; Heim, W.; Berlin, J.; Baron, A.; Griffing, S.; Holmgren, E.; et al. Bevacizumab plus Irinotecan, Fluorouracil, and Leucovorin for Metastatic Colorectal Cancer. N. Engl. J. Med. 2004, 350, 2335-2342. [CrossRef]

11. Loupakis, F.; Antonuzzo, L.; Bachet, J.-B.; Kuan, F.-C.; Macarulla, T.; Pietrantonio, F.; Xu, R.-H.; Taniguchi, H.; Winder, T.; Yuki, S.; et al. Practical considerations in the use of regorafenib in metastatic colorectal cancer. Ther. Adv. Med Oncol. 2020, 12. [CrossRef]

12. Van Cutsem, E.; Tabernero, J.; Lakomy, R.; Prenen, H.; Prausová, J.; Macarulla, T.; Ruff, P.; Van Hazel, G.A.; Moiseyenko, V.; Ferry, D.; et al. Addition of Aflibercept to Fluorouracil, Leucovorin, and Irinotecan Improves Survival in a Phase III Randomized Trial in Patients with Metastatic Colorectal Cancer Previously Treated With an Oxaliplatin-Based Regimen. J. Clin. Oncol. 2012, 30, 3499-3506. [CrossRef]

13. Chau, I.; Fakih, M.; García-Alfonso, P.; Linke, Z.; Casado, A.R.; Marques, E.P.; Picard, P.; Celanovic, M.; Cartwright, T. Safety and Effectiveness of Aflibercept + Fluorouracil, Leucovorin, and Irinotecan (FOLFIRI) for the Treatment of Patients with Metastatic Colorectal Cancer (mCRC) in Current Clinical Practice: OZONE Study. Cancers 2020, 12, 657. [CrossRef] [PubMed]

14. Ivanova, J.I.; Saverno, K.R.; Sung, J.; Duh, M.S.; Zhao, C.; Cai, S.; Vekeman, F.; Peevyhouse, A.; Dhawan, R.; Fuchs, C.S. Real-world treatment patterns and effectiveness among patients with metastatic colorectal cancer treated with ziv-aflibercept in community oncology practices in the USA. Med. Oncol. 2017, 34, 193. [CrossRef] [PubMed]

15. Buchler, T.; Kiss, I.; Hornova, J.; Fiala, O.; Wiesnerova, M.; Svoboda, M.; Silar, J.; Kopeckova, K.; Poprach, A.; Finek, J.; et al. Sequential Treatment with Bevacizumab and Aflibercept for Metastatic Colorectal Cancer in Real-World Clinical Practice. Target. Oncol. 2020, 15, 193-201. [CrossRef] [PubMed]

16. Vera, R.; Mata, E.; González, E.; Juez, I.; Alonso, V.; Iranzo, P.; Martínez, N.P.; Lopez, C.L.; Cabrera, J.M.; Safont, M.J.; et al. Is aflibercept an optimal treatment for wt RAS mCRC patients after progression to first line containing anti-EGFR? Int. J. Color. Dis. 2020, 35, 739-746. [CrossRef]

17. Feliu, J.; De Corcuera, I.D.; Manzano, J.L.; Valladares-Ayerbes, M.; Alcaide, J.; García, J.A.; Vera, R.; Sastre, J. Effectiveness and safety of aflibercept for metastatic colorectal cancer: Retrospective review within an early access program in Spain. Clin. Transl. Oncol. 2016, 19, 498-507. [CrossRef]

18. Yusof, M.M.; Abdullah, N.M.; Sharial, M.M.; Zaatar, A. Safety and Management of Toxicity Related to Aflibercept in Combination with Fluorouracil, Leucovorin and Irinotecan in Malaysian Patients with Metastatic Colorectal Cancer. Asian Pac. J. Cancer Prev. 2016, 17, 973-978. [CrossRef]

19. Montes, A.F.; Lago, N.M.; Rúa, M.C.; Gómez, J.D.L.C.; Villaroel, P.G.; Méndez, J.C.M.; Fernández, M.J.; Fernández, M.S.; López, M.R.; Aldana, G.Q.; et al. Efficacy and safety of FOLFIRI/aflibercept in second-line treatment of metastatic colorectal cancer in a real-world population: Prognostic and predictive markers. Cancer Med. 2019, 8, 882-889. [CrossRef] [PubMed]

20. Kim, J.; Kim, H.; Hong, J.Y.; Lee, J.; Park, S.H.; Park, J.O.; Park, Y.S.; Lim, H.Y.; Kang, W.K.; Kim, S.T. Prognostic Factors of Survival with Aflibercept and FOLFIRI (fluorouracil, leucovorin, irinotecan) as Second-line Therapy for Patients with Metastatic Colorectal Cancer. J. Cancer 2021, 12, 460-466. [CrossRef] [PubMed]

21. Chong, D.Q.; Manalo, M.; Imperial, M.; Teo, P.; Yong, G.; Ng, M.; Tan, I.B.; Choo, S.P.; Chua, C. Safety and efficacy of aflibercept in combination with fluorouracil, leucovorin and irinotecan in the treatment of Asian patients with metastatic colorectal cancer. Asia-Pac. J. Clin. Oncol. 2016, 12, 275-283. [CrossRef] [PubMed]

22. Ottaiano, A.; Capozzi, M.; Tafuto, S.; De Stefano, A.; De Divitiis, C.; Romano, C.; Avallone, A.; Nasti, G. Folfiri-Aflibercept vs. Folfiri-Bevacizumab as Second Line Treatment of RAS Mutated Metastatic Colorectal Cancer in Real Practice. Front. Oncol. 2019, 9, 766. [CrossRef]

23. Auvray, M.; Tougeron, D.; Auclin, E.; Moulin, V.; Artru, P.; Hautefeuille, V.; Hammel, P.; LeComte, T.; Locher, C.; Sickersen, G.; et al. Efficacy and Safety of Aflibercept in Combination with Chemotherapy Beyond Second-Line Therapy in Metastatic Colorectal Carcinoma Patients: An AGEO Multicenter Study. Clin. Color. Cancer 2020, 19, 39-47.e5. [CrossRef]

24. Common Terminology Criteria for Adverse Events (CTCAE) v4.0 (CTCAE v4.03). Available online: https:/ / evs.nci.nih.gov/ftp1 /CTCAE/CTCAE_4.03/CTCAE_4.03_2010-06-14_QuickReference_5x7.pdf (accessed on 26 May 2021).

25. Eisenhauer, E.A.; Therasse, P.; Bogaerts, J.; Schwartz, L.; Sargent, D.; Ford, R.; Dancey, J.; Arbuck, S.; Gwyther, S.; Mooney, M.; et al. New response evaluation criteria in solid tumours: Revised RECIST guideline (version 1.1). Eur. J. Cancer 2009, 45, $228-247$. [CrossRef] 
26. Tabernero, J.; Van Cutsem, E.; Lakomý, R.; Prausová, J.; Ruff, P.; van Hazel, G.A.; Moiseyenko, V.; Ferry, D.R.; McKendrick, J.J.; Soussan-Lazard, K.; et al. Aflibercept versus placebo in combination with fluorouracil, leucovorin and irinotecan in the treatment of previously treated metastatic colorectal cancer: Prespecified subgroup analyses from the VELOUR trial. Eur. J. Cancer 2014, 50, 320-331. [CrossRef]

27. Abrams, T.A.; Meyer, G.; Schrag, D.; Meyerhardt, J.A.; Moloney, J.; Fuchs, C.S. Chemotherapy Usage Patterns in a US-Wide Cohort of Patients With Metastatic Colorectal Cancer. J. Natl. Cancer Inst. 2014, 106, djt371. [CrossRef]

28. Park, H.S.; Chun, Y.J.; Kim, H.S.; Kim, J.H.; Lee, C.-K.; Beom, S.-H.; Shin, S.J.; Ahn, J.B. Clinical features and KRAS mutation in colorectal cancer with bone metastasis. Sci. Rep. 2020, 10, 21180. [CrossRef] [PubMed]

29. Nozue, M.; Oshiro, Y.; Kurata, M.; Seino, K.-I.; Koike, N.; Kawamoto, T.; Taniguchi, H.; Todoroki, T.; Fukao, K. Treatment and prognosis in colorectal cancer patients with bone metastasis. Oncol. Rep. 2002, 9, 109-112. [CrossRef] [PubMed]

30. Maiello, E.; Pomella, V.; Wirapati, P.; Di Bartolomeo, M.; Zampino, M.; Leone, F.; Latiano, T.; Pietrantonio, F.; Ravenda, P.; Marino, D.; et al. Aflibercept efficacy according to sidedness, RAS and BRAF mutations. Findings from the VELOUR trial in second line therapy of advanced colorectal cancer patients. Ann. Oncol. 2017, 28, vi7. [CrossRef]

31. Riechelmann, R.P.; Srimuninnimit, V.; Bordonaro, R.; Kavan, P.; Di Bartolomeo, M.; Maiello, E.; Cicin, I.; García-Alfonso, P.; Chau, I.; Fedyanin, M.Y.; et al. Aflibercept Plus FOLFIRI for Second-line Treatment of Metastatic Colorectal Cancer: Observations from the Global Aflibercept Safety and Health-Related Quality-of-Life Program (ASQoP). Clin. Color. Cancer 2019, 18, 183-191.e3. [CrossRef] [PubMed]

32. Bennouna, J.; Sastre, J.; Arnold, D.; Österlund, P.; Greil, R.; Van Cutsem, E.; von Moos, R.; Viéitez, J.M.; Bouché, O.; Borg, C.; et al. Continuation of bevacizumab after first progression in metastatic colorectal cancer (ML18147): A randomised phase 3 trial. Lancet Oncol. 2013, 14, 29-37. [CrossRef]

33. Masi, G.; Salvatore, L.; Boni, L.; Loupakis, F.; Cremolini, C.; Fornaro, L.; Schirripa, M.; Cupini, S.; Barbara, C.; Safina, V.; et al. Continuation or reintroduction of bevacizumab beyond progression to first-line therapy in metastatic colorectal cancer: Final results of the randomized BEBYP trial. Ann. Oncol. 2015, 26, 724-730. [CrossRef] [PubMed]

34. Alcalay, A.; Wun, T.; Khatri, V.; Chew, H.K.; Harvey, D.; Zhou, H.; White, R.H. Venous Thromboembolism in Patients with Colorectal Cancer: Incidence and Effect on Survival. J. Clin. Oncol. 2006, 24, 1112-1118. [CrossRef] [PubMed]

35. Zangari, M.; Fink, L.M.; Elice, F.; Zhan, F.; Adcock, D.M.; Tricot, G.J. Thrombotic Events in Patients with Cancer Receiving Antiangiogenesis Agents. J. Clin. Oncol. 2009, 27, 4865-4873. [CrossRef] [PubMed] 